Journal of the Magnetics Society of Japan Vol. 15 Supplement, No. S2 (1991)

(c) 1991 by The Magnetics Society of Japan

\title{
RELIABILITY IN QUASI-CONTACT HEAD/DISK INTERFACE
}

\author{
Haruyuki MORITA, Kazumasa FUKUDA and Joichiro EZAKI \\ New Product Development Division, TDK CORPORATION \\ 543 Otai, Saku-shi, Nagano 389-02, Japan
}

\begin{abstract}
A simple thin film head which we call "Mellisuga Head" has' been developed. The head has an extremely low flying height, which can be adjusted between $0.03 \mu \mathrm{m}$ and $0.07 \mu \mathrm{m}$ at $11.3 \mathrm{~m} / \mathrm{s}$ of head/disk relative velocity by modifying the gross ABS area and a gram load. Significant improvement of electrical performance was attained primarily due to the extremely low flying height. Then, the reliability in the contact start stop (CSS) process and the soft dynamic load/unload (SDL) process of the head on two types of thin film disk was investigated. In the Mellisuga Head operation on a $\gamma-\mathrm{Fe}_{2} \mathrm{O}_{3}$ disk with a glass substrate, 50,000 CSS cycles as well as 100,000 SDI cycles were completed successfully. This confirms that the $\gamma-\mathrm{Fe}_{2} \mathrm{O}_{3}$ disk has an especial advantage to the Mellisuga Head operation. It was also shown that the head could be operated on a metal disk with a glass substrate during the SDI process.
\end{abstract}

\section{INTRODUCTION}

Magnetic recording heads in rigid disk drives tend to be miniaturized because small-sized head sliders have the advantages of better slider traceability to external fluctuation and better slider stability [1]. As one of the small-sized head sliders, we have proposed a very simple magnetic recording head - we call it "Mellisuga Head" - which is entirely different from a 3380 type two-rail taper flat slider[2],[3].

The Mellisuga Head easily achives very low flying heights. It is possible, however, that the tribological problems in load/unload process may be raised by the low flying heights.

In this report, the method of a flying height control and the read/write performance in extremely low flying height are discussed. The reliability in the contact start stop (CSS) process and soft dynamic load/unload (SDL) process of the Mellisuga Head on two types of thin film disk is also described.

\section{EXPERIMENTAL PROCEDURE}

\section{Mellisuga Head}

An underside view of the newly developed Mellisuga Head is shown in Fig. 1 together with the 3380 type slider. The
Mellisuga Head is characterized by having a single, almost square and flat air bearing surface (ABS). The simple geometrical structure of the head is another advantage from the viewpoint of an easy manufacturing process. The size of the head is approximately $30 \%$ of the 3380 type slider.

Pole dimensions of the head element were arranged to be $2.4 \mu \mathrm{m}$ in leading pole thickness, $0.32 \mu \mathrm{m}$ in gap Iength and $2.3 \mu \mathrm{m}$ in trailing pole thickness. Track width and number of coil turns were adjusted to $12.7 \mu \mathrm{m}$ and 32 turns, respectively. The gram load of the head suspension was $6.5 \mathrm{~g}$ and the slider material was $\mathrm{Al}_{2} \mathrm{O}_{3}-\mathrm{TiC}$.

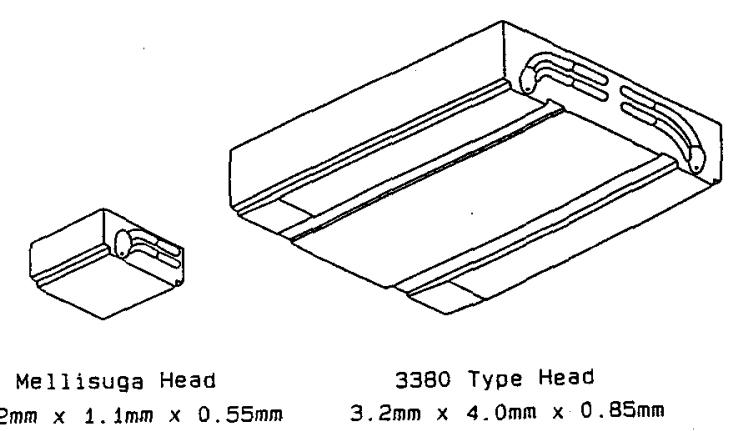

Fig.1. Underside views of Mellisuga Head and 3380 type head. 
Two-rail taper flat sliders of $70 \%$ type were also prepared from the same wafer for reference. Its slider width was $380 \mu \mathrm{m}$ and the gram load was $4.0 \mathrm{~g}$.

Flying height was measured on silica glass disk by using a flying height tester. Read/write performance was tested by using a read/write, analyzer and spin-stand combination. Write current was adjusted to $30 \mathrm{mAo}-\mathrm{p}$, and the cut-off frequency of the read channel was set at $15 \mathrm{MHz}$ at $-3 \mathrm{~dB}$ point.

Touch-down velocity of the Mellisuga Head on a spinning disk surface was measured from the dynamic friction force versus head/disk relative velocity plot. The touch-down velocity is defined by the head/disk relative velocity at which the rubbing between the head and the spinning disk arises in a deceleration process of the disk.

\section{Disk}

The aluminum substrate for the popularly used metal thin film disk is usually texturized to produce a magnetic orientation in magnetic layer and a certain magnitude of disk surface roughness. Consequently, the disk surface roughness Rmax becomes $0.07 \mu \mathrm{m}$ to $0.1 \mu \mathrm{m}$. This value is too large to operate the Meliisuga Head. As the flying height of the Mellisuga Head is extremely low, the surface roughness Rmax of the disk to be employed must be less than $0.05 \mu \mathrm{m}$. The following two types of disk were used.

(1) Sputtered $\gamma-\mathrm{Fe}_{2} \mathrm{O}_{3}$ thin film disk. $\gamma-\mathrm{Fe}_{2} \mathrm{O}_{3}$ layer is formed on a smooth glass substrate and the disk does not have any protective overcoat layers.

(2) Sputtered metal thin film disk. $\mathrm{Cr}$ layer, $\mathrm{Co}-\mathrm{Ni}-\mathrm{Cr}$ layer and carbon overcoat layer are sputtered on a smooth glass substrate.

Very thin liquid lubricant was coated less than $20 \mathrm{~A}$ on the both type disks. Surface roughness Rmax of the disk were measured with a contact surface profizometer which has a $0.2 \mu \mathrm{m} \times 0.2 \mu \mathrm{m}$ pyramid-shaped stylus.

\section{CSS Process}

A CSS cycle consists of 5 seconds acceleration to $11.3 \mathrm{~m} / \mathrm{s}$ of head/disk relative velocity, 5 seconds dwell, 5 seconds deceleration and 5 seconds stop. The CSS cycle was repeated maximum 50,000 times.

\section{SDI Process}

Fig.2 shows a SDL procedure schematically [4]. A triangular-shaped ramp which runs along a longitudinal axis defined by the head/arm assembly is formed on a suspension arm face. In the unloading stage, the triangular-shaped ramp is ridden against the stationary cam, resulting the Mellisuga Head leaving the spinning disk (Fig.2(a)). In the case of the head loading, as the suspension arm moves towards the spinning disk, the ramp passes over the cam, then the head is gradually approached to the spinning disk surface (Fig.2(b)). Following the loading, the head is seeked to the innermost radius portion on the spinning disk surface (Fig.2(c)).

SDL process was carried out by repeating the cycle of $(a)-(b)-(c)-(b)-(a)$ 100,000 times.

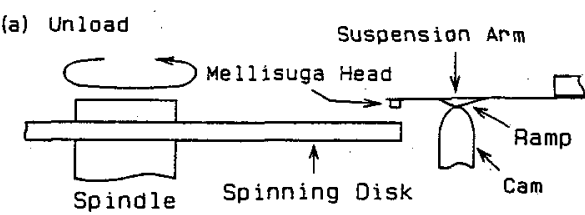

(b) Load

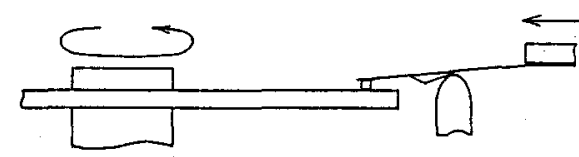

(c) Seek to Innermost

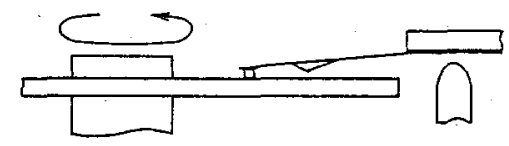

Fig.2. SDL procedure. 


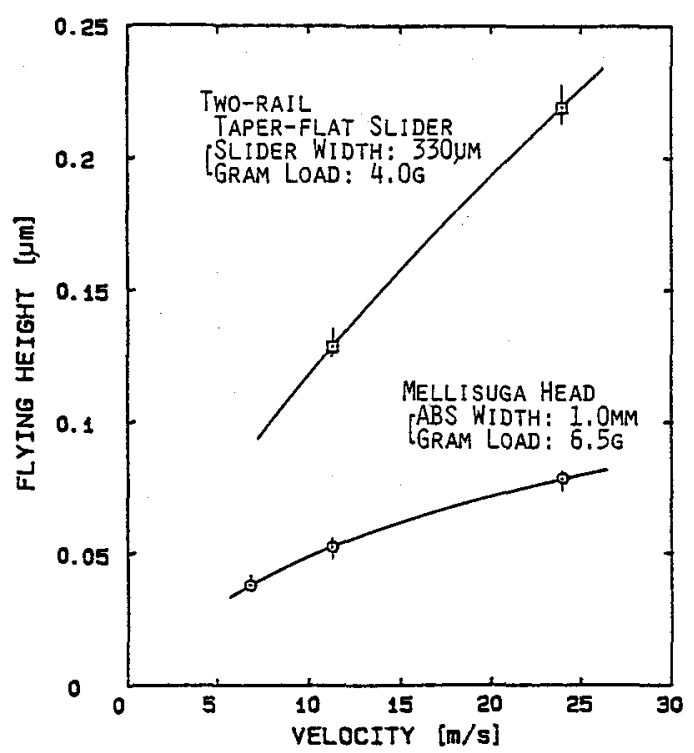

Fig.3. Dependence of flying height on head/disk relative velocity in Mellisuga Head and two-rail taper flat slider.

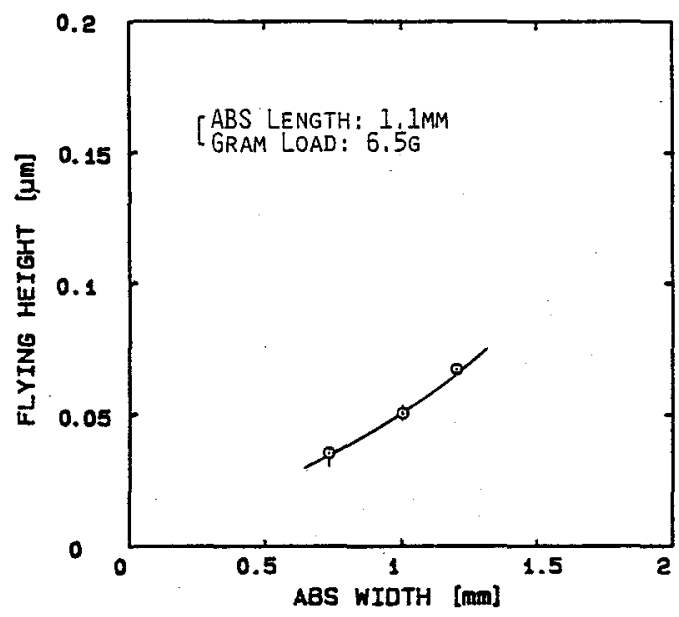

Fig.4. Relationship between ABS width and flying height in Mellisuga Head.

\section{RESULTS AND DISCUSSION}

Flying Height

Fig. 3 shows the relationship between the flying height and the head/disk relative velocity of the Mellisuga Head and the conventional two-rail taper flat slider. The ABS width of the Mellisuga Head was adjusted to $1.0 \mathrm{~mm}$ by modifying the width of the shallow slots on the both sides of the ABS. As shown in Fig.3, the flying height of the Mellisuga Head is extremely low, depending on the gross ABS area and the gram load.

The relationship between the flying height at $11.3 \mathrm{~m} / \mathrm{s}$ and the $A B S$ width of the Mellisuga Head is shown in Fig.4, where the ABS length and the gram load are kept constant at $1.1 \mathrm{~mm}$ and $6.5 \mathrm{~g}$, respectively. This figure shows that the flying height of the head can be successfully adjusted in almost the same manner as conventional two-rail taper flat sliders. The flying height of the head can also be adjusted by modifying the gram load and the edge blend of the ABS.

It must be emphasized that the amount of scatter in flying heights is small enough within the new head samples produced under same conditions. The variation of the flying heights was within $\pm 10 \%$ and almost comparable with the conventional sliders. This confirms that the Mellisuga Head is controllable and stable as far as aerodynamic performance is concerned.

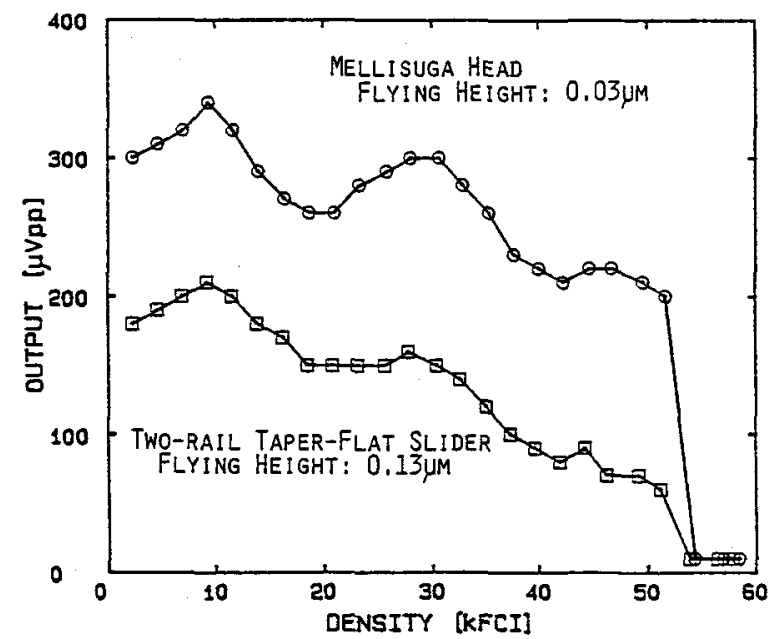

Fig.5. Bit density responses of Mellisuga Head with $0.03 \mu \mathrm{m}$ flying height and tworail taper flat slider with $0.13 \mu \mathrm{m}$ flying height. 
Read/write Performance

Read/write performance of the Mellisuga Head was measured on $\gamma-\mathrm{Fe}_{2} \mathrm{O}_{3}$ disk with coercivity of $1200 \mathrm{Oe}$, remanence of $2500 \mathrm{C}$, squareness ratio of 0.70 and thickness of $1000 \mathrm{~A}$. Bit density response at $11.3 \mathrm{~m} / \mathrm{s}$ of the Mellisuga Head with a flying height of $0.03 \mu \mathrm{m}$ is compared with two-rail taper flat slider with a flying height of $0.13 \mu \mathrm{m}$ in Fig.5. Significant improvement in read/write performance. was attained due to the extremely low flying height.

\section{Surface Roughness}

The surface roughness $R \max$ of both types of thin film disk used are shown in Table I, including the other results. Since the disks employ carefuliy smoothed glass substrates, the surface roughnesses are extremely improved in comparison to ordinary disks with aluminum substrates.

\section{Touch-Down Velocity}

Fig.6 shows an example of touch-down characteristics of the Mellisuga Head with a flying height of $0.05 \mu \mathrm{m}$. This characteristics were measured "on the $y-\mathrm{Fe}_{2} \mathrm{O}_{3}$ disk surface with a surface roughness Rmax of $160 \mathrm{~A}$. Although the figure shows the touch-down velocity of $3.2 \mathrm{~m} / \mathrm{s}$, the velocity decreases almost linearly with the decreasing surface roughness of the disk as shown in Fig.?.

\section{CSS Process}

The CSS results in a combination of the Mellisuga Head and the two types of disk are shown in Table I. In the investigations of the CSS process and the following SDI process, the Mellisuga Head with a flying height of 0.05 um was used.

on the $\gamma-\mathrm{Fe}_{2} \mathrm{O}_{3}$ disks," 50,000 CSS cycles were performed with no failure if the surface roughnesses of the disks were adjusted more than or equal to a $160 \mathrm{~A}$.

The CSS cycles were carried out under the severe CSS condition, where the head having an extremely low flying height and no taper on the ABS was employed. The main factor for the success in this severe CSS process is thought to be the intrinsic high hardness of the $\gamma-\mathrm{Fe}_{2} \mathrm{O}_{3}$ magnetic layer.

From the result of Fig.7, the time length that the head rubs the disk surface

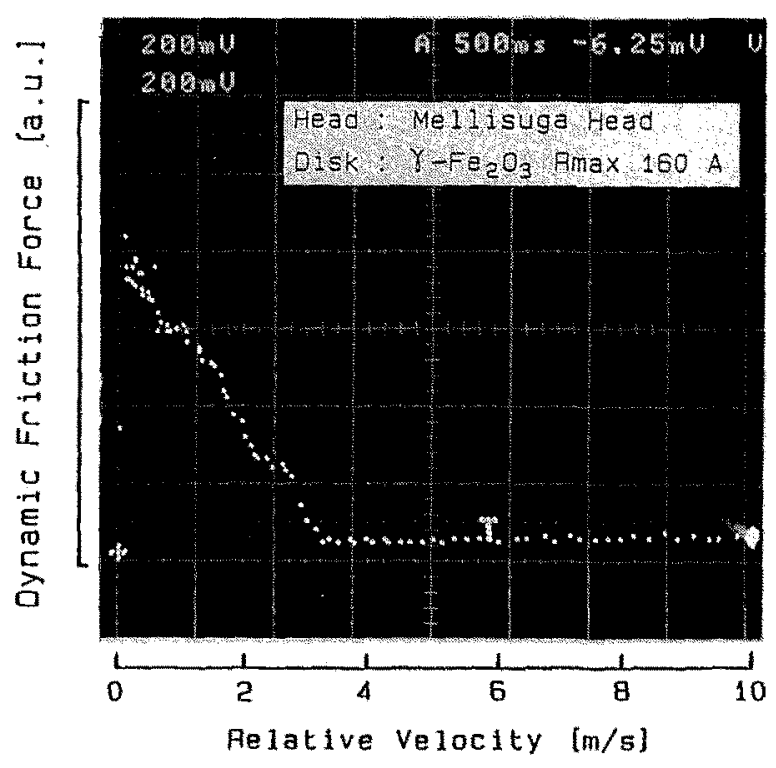

Fig.6. Touch-down characteristics of Mellisuga Head.

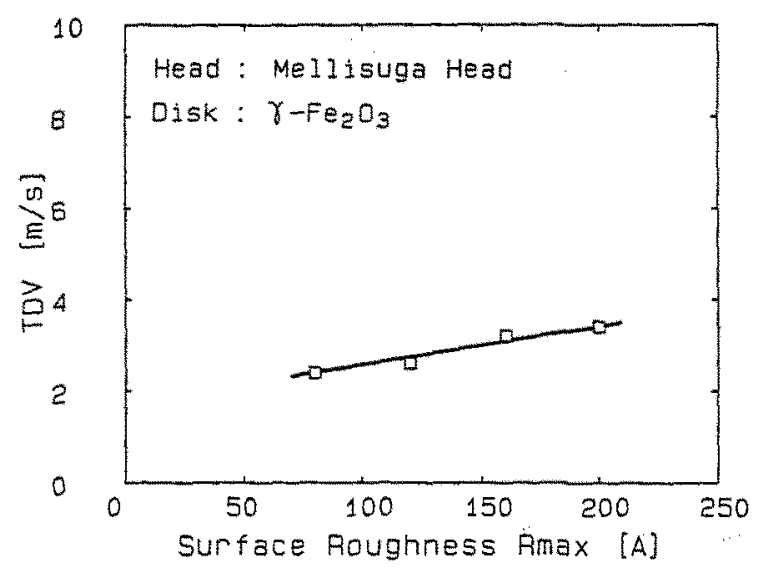

Fig.7. Dependence of touch-down velocity on disk surface roughness Rmax.

in a CSS cycle becomes shorter with the decrease of the surface roughness, and the CSS process is considered to be executed more easily with the decreasing roughness. In the CSS process using disks having extremely small surface roughnesses ( $80 \mathrm{~A}$, $120 \mathrm{~A}$ ), however, there arises the problem of head-to-disk stiction. 
Table I Surface roughness and reliability results.

\begin{tabular}{|c|c|c|c|}
\hline Disk & $\operatorname{Rmax}(A)$ & CSS & SDL \\
\hline$\gamma-\mathrm{Fe}_{2} \mathrm{O}_{3}$ & $\begin{array}{r}80 \\
120 \\
160 \\
200\end{array}$ & $\begin{array}{l}\text { Sticking from start } \\
\text { Sticking in } 5,000 \text { cycles } \\
50,000 \text { cycles completed } \\
50,000 \text { cycles completed }\end{array}$ & $\begin{array}{c}100,000 \text { cycles completed } \\
100,000 \text { cycles completed } \\
\text { - } \\
100,000 \text { cycles completed }\end{array}$ \\
\hline Metal & $\begin{array}{l}110 \\
380\end{array}$ & $\begin{array}{l}\text { Crash in } 1200 \text { cycles } \\
\text { Crash in } 2100 \text { cycles }\end{array}$ & $\begin{array}{l}100,000 \text { cycles completed } \\
\text { Many short and slight } \\
\text { scratches in } 100,000 \text { cycles }\end{array}$ \\
\hline
\end{tabular}

On the other hand, the metal disks were crashed in the CSS process regardless of the surface roughness. The use of the Mellisuga Head in the CSS process is thought to be severe on the metal aisks, which have moderate hardness of magnetic and overcoat layers.

SDL Process

SDL tests were carried out on the three $\gamma-\mathrm{Fe}_{2} \mathrm{O}_{3}$ disks and the two metal disks, and the results are shown in Table $I$. on the $\gamma-\mathrm{Fe}_{2} \mathrm{O}_{3}$ disks, it was found that 100,000 times of SDI cycle were completed successfully. Stiction, which was a problem in the CSS process on the disks having too smooth surface, did not have an effect on the SDI process.

On the metal disks which did not have sufficient CSS durability, head crash or critical surface wear of the disk did not take place in 100,000 times of SDL cycle. On the metal disk having the surface roughness of $380 \mathrm{~A}$, however, many short and slight scratches were noticed on its surface. The scratches are thought to have been brought during the head seek motion.

As the Mellisuga Head has an extremely low flying height, the head in the loading process is thought to produce a contact with the spinning disk surface to some extent. Contact time is very short in the SDI process, stiction on the extremely smooth surface as well as the moderate hardness of the metal disk is not thought to cause fatal damage to the head/disk interface.
From these results, the surface roughness Rmax of the disk is considered to be one of the important factors in the SDL process with the Mellisuga Head. The roughness should be adjusted less than 300 A or $200 \mathrm{~A}$ in order to avoid head contact with the fine irregularity on the disk surface.

\section{CONCLUSTON}

The newly developed Meliisuga Head has a simple structure and an extremely low flying height.

The Mellisuga Head was found to show enough stability and reliability both in aerodynamic and read/write performance at extremely low flying heights.

For the Mellisuga Head operation, the employment of the disk with a carefully smoothed glass substrate is recommended, and the prefered disk surface roughness Rmax is less than $300 \mathrm{~A}$ or $200 \mathrm{~A}$.

Specifically, the usage of $\gamma^{-F_{2}} \mathrm{O}_{3}$ thin film disks with smooth glass substrates is advantageous from the point of view that the Mellisuga Head can be loaded and unloaded on the disk in the CSS process as well as in the SDL process. In the SDL process, the head can also be operated on a metal disk with a smooth glass substrate.

These results suggest that the head will be applied to near future rigid disk drives including perpendicular magnetic recording drives. 


\section{ACKNOWLEDGMENTS}

The authors would like to express their thanks to President H. Satoh, Executive Managing Director T. Masaki and Managing Director 0 . Wada of TDK CORPORATION for their encouragement and support of this work.

\section{REFERENCES}

[1] C. A. Briggs and F. E. Talke, "Investigation of the dynamics of $50 \%$ sliders using laser doppler vibrometry", IEEE Trans. Magn., vol.26, pp.3027-3032, 1990.

[2] H. Morita, A. Nakamura and J. Ezaki, "Reliability in load/unload process of new type slider", to be published in IEEE Trans. Magn. Vol.27, No.6.

[3] K. Fukuda, H. Hamanaka, A. Sasaki and J. Ezaki, "Thin film head for quasicontact head/disk interface", to be published in IEEE Trans. Magn., Vol.27, No. 6 .

[4] K. M. Anderson and J. H. Morehouse, U. S. Patent 4,535,374, 1985. 